\title{
Research of Improved Dijkstra Algorithm Based Park Logistics Scheduling
}

\author{
Mo Taiping ${ }^{*}, 1$, Duan Renhang ${ }^{2}$ and Zhao Peisi ${ }^{2}$ \\ ${ }^{I}$ School of Mechano-electronic Engineering, Xidian University, Xi'an 710071, China; ${ }^{2}$ School of Electronic Engineering \\ \& Automation, Guilin University of Electronic Technology, Guilin 541004, China
}

\begin{abstract}
In the research of the park gathering industry manufacturing mode, the route of existing systems to provide logistics and transportation services often cannot meet the actual scheduling needs. To find a suitable distribution method is necessary. In this paper, based on the background of the industrial park logistics scheduling, the Dijkstra algorithm is investigated and proposed an improved algorithm based on the original algorithm. Experimental results show that the improved Dijkstra algorithm not only retains the advantages of the original algorithm, but also can quickly and accurately find out the optimal delivery route based on the latest traffic conditions, significantly improve the logistics park path search efficiency and optimize the quality of the scheduling. Realized reduce consumption, improve efficiency and reduce the purposes of logistics costs throughout the park.
\end{abstract}

Keywords: Park logistics scheduling, Dijkstra algorithm, optimal path, impact factor.

\section{INTRODUCTION}

During the 30-year reform and opening up, especially since the nineties. Each area of our country established various types of industrial park according to the characteristic of themselves and the original industrial base structure. These industrial parks are required to enhance their core competitiveness to attract money through complete and perfect service facilities. Park Logistics is an important aspect of supporting services, which can help companies link the park to better achieve its own supply chain management, resource conservation costs and improve the attractiveness of the park. Therefore, use of appropriate logistics scheduling method in the park areas to the park can improve the efficiency of logistics and distribution, and improve the competitiveness of the industrial park [1].

Now, due to the industrial park of our country started relatively late and the special geographical location of some parks, and the weakness of the enterprise foundation, the park logistics service is basically in the state of disorder, in another way, logistics movement is only the enterprise's own behavior, industrial park has not played its corresponding action and its distribution pattern is not quite informal. So the logistics network optimization and the route planning logistics path have become a very critical issue to be faced by the logistics park. These efforts whether can proceed smoothly depend on the reasonableness of the logistics park mode scheduling method. Proper scheduling can help enterprises in the park to achieve its own supply chain management better, resource conservation costs and improve the attractiveness of the park.

Therefore, the study of patterns of distribution algorithm on the park mode has significant economic value and significance.

\section{PARK LOGISTICS SCHEDULING}

For Industrial Park, Logistics rationalization at the level of park refers to the study of integration, allocation and utilization of regional logistics resources. It also refers to the research of the influence of the negative externalities on enterprise logistics development caused by the one-sided pursuit of efficiency under the decentralized logistics resources management from an overall perspective. Logistics integration is an important objective of the research. The so-called logistics integration is to get the system running logistics scale and intensive, by common, socialization logistics collaborative way, which is an essential goal of the regional logistics system establishes. The implementation of logistics integration is the impact on the efficiency of regional logistics system produced in the process of an enterprise or supply chain logistics efficient pursuit of the self-organization optimization [2].

Reasonable transportation plan, is not unilateral pursuit of optimization index, such as to search the shortest transport distance, to pursue the cheapest cost, the fastest speed, or the minimum intermediate links. Transport links to each shift factors influence each other and only the coordination of various factors can optimize the transport. According to the customer's requirements on time, cost, distance, and characteristics of goods to design reasonable transport routes, transport tools and transit links.

This paper introduces Dijksta algorithm, then to study the algorithm and reveal the limitations of the algorithm, propose a new optimization algorithm based on this algorithm. The improved Dijkstra algorithm obtained the most suitable distribution path according to the real-time traffic conditions on logistics scheduling and distribution road. So as to further achieve the purpose of a reasonable dispatch of modern logistics industrial park, and increasing the competitiveness of the industrial park. 


\section{TRADITIONAL DIJKSTRA ALGORITHM}

The existing logistics and transport services in the public system cannot meet the actual travel route travel needs of the people, due to a variety of technical reasons. It is necessary to find a way to optimize logistics and distribution, to meet the minimum cost. To identify the specific source point to the end of the optimal delivery route, this article needs to relate to the problem of searching the shortest paths.

The algorithm that used for solving the shortest path problem algorithm is called "the shortest path algorithm" [3]. The monophyletic shortest path has a universal significance and exists many algorithms, such as depth first search algorithm, the shortest path algorithm, the dynamic programming, Dijkstra algorithm, the biggest related edge method, the biggest relevant points, $\mathrm{A} *$ algorithm and SPFA algorithm [4].

\subsection{Algorithm Introduction}

Currently, to find a shortest path, Dijkstra algorithm is a good algorithm. The algorithm is simple, easy to implement. Its basic idea is to follow the path length distance from the starting point of the order to produce the shortest path.

The algorithm was proposed in 1959 by the Dutch mathematician E.W.Dijkstra. It is known as Dijkstra algorithm. The algorithm is a typical single-source shortest path algorithm for computing the shortest path from one node to all other nodes. Its main feature is the starting point as the center point outward extension until extend to the end point. The algorithm applied the greedy algorithm model. It is now recognized as a perfect way to solve the shortest path.

\subsection{Dijkstra Algorithm Processes}

The purpose of Dijkstra algorithm is given a directed network $D=(V, A, W)$, any $\operatorname{arc} a_{i j} \in \mathrm{A}$, defined a right Weight $w\left(a_{i j}\right)=w_{i j}$.

Assume that $\mathrm{D}$ has two vertices $v_{s}$ and $v_{\mathrm{t}}$.In all of the path from $v_{s}$ to $v_{\mathrm{t}}$, find a path $\mathrm{P}^{\prime} w\left(P^{\prime}\right)=\min _{\mathrm{P}} w(\mathrm{P})$, Weights path $\mathrm{P}^{\prime}$ is the distance $v_{s}$ to $v_{\mathrm{t}}$, that is $u_{\mathrm{st}}$. It is the shortest path between two vertices [5].

Specific steps are as follows:

The first step:

First find a directed network

vertices $v_{s}$ to $D=(V, A, W)$ in the shortest length of all the other vertices. The calculated path length are arranged in order of short to long: $u_{0} \leq u_{1} \leq \mathrm{L} \leq u_{n}, u_{0}$ represents the length $v_{s}$ to $v_{s}$.

The corresponding shortest path is $P_{0}, P_{1}, \mathrm{~L}, P_{\mathrm{n}}$.

$$
\begin{aligned}
& \mathrm{X}_{0}=\left\{v_{s}\right\} \\
& \overline{\mathrm{X}}_{0}=\mathrm{VX}_{0} \\
& u_{0}=0 \\
& u_{j}=w_{s j}(1 \leq j \leq n) \\
& X_{k}=\left\{v_{s}\right\}
\end{aligned}
$$

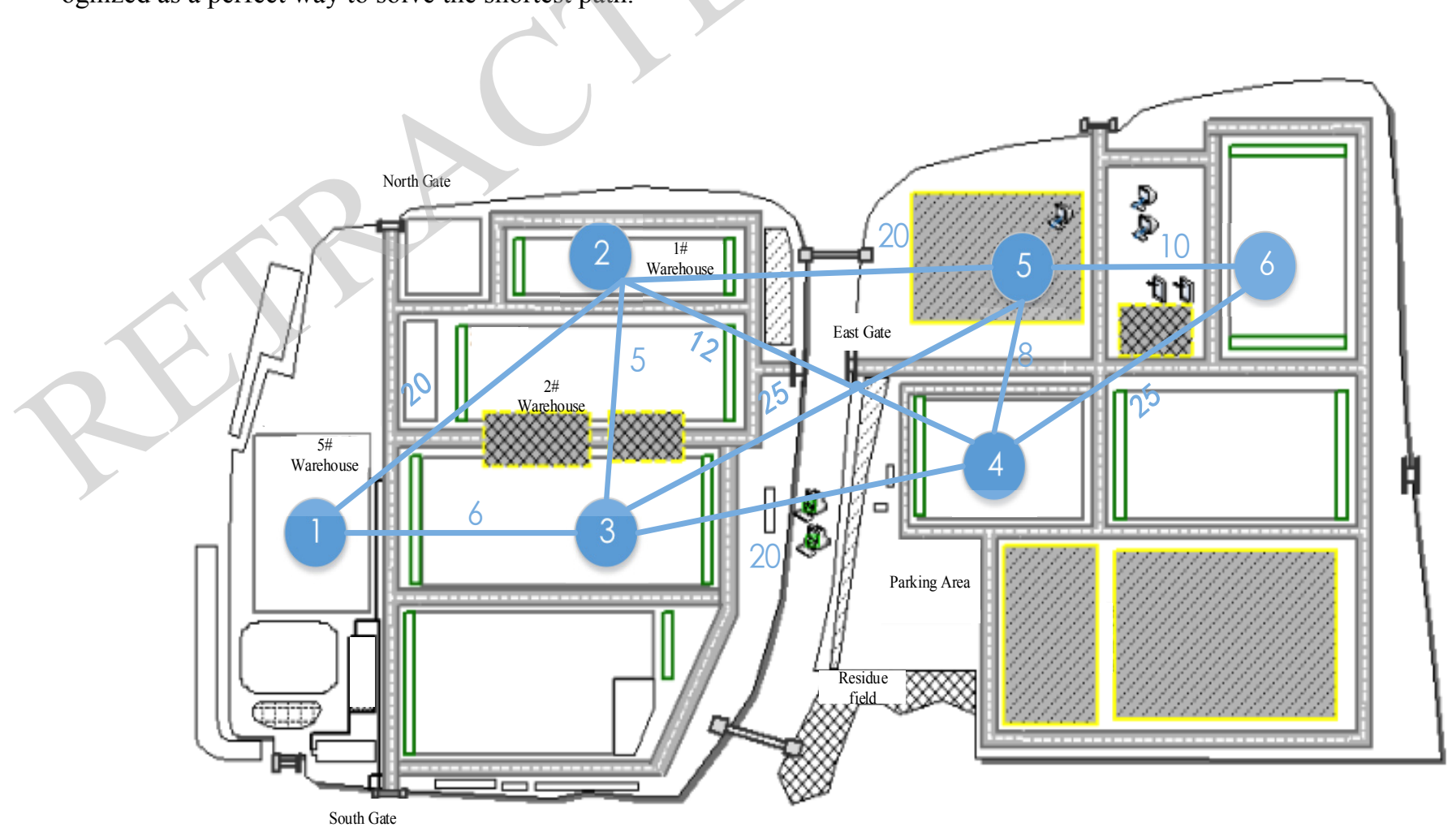

Fig. (1). Industrial park logistics delivery map. 
$\overline{X_{k}}=V \backslash X_{k}$

The initial state is:

$k=0$

$\lambda_{i}=0(0 \leq i \leq n)$

where,

$X_{k}$---the set of point;

$v_{s}$--- the starting point;

$V$--- the set of all points;

$u_{j}$--- the distance between the starting point;

$w_{i j}$--- the weights between two points;

$\lambda_{j}$--- the previous point label;

$i, j, k---$ the parameters.

If $\lambda_{j}=m$, it means the point in front of the $v_{j}$ is $v_{m}$.

Step 2:

$$
u_{1}=\min _{v_{i} \in \overline{\mathrm{X}}_{0}}\left\{w_{s i}\right\}
$$

Find the nearest point from the starting point in set $\overline{\mathrm{X}}_{0}$. The point which is obtained is $v_{1}$.

$P_{1}=P\left(v_{\mathrm{s}}, v_{1}\right)$

where,

$v_{1}$--- the nearest point from the starting point in set $\overline{\mathrm{X}}_{0}$.

Then go to the 3rd step.

Step 3:

Take $v_{1}$ as an intermediate point, find the closest point to $v_{1}$ in the set $\overline{X_{k+1}}$.

$X_{k+1}=X_{k} \mathrm{U}\left\{v_{1}\right\}$

$\overline{X_{k+1}}=\bar{X}_{k} \backslash\left\{v_{1}\right\}$

After getting the point which is nearest from $v_{1}$, then $\mathrm{k}$ add 1 , find new intermediate point, repeat operations.

$u_{k+1}=\min _{\substack{v_{i} \in \mathrm{X}_{k} \\ v^{\prime} \in \overline{\mathrm{X}}_{k}}}\left\{u_{\mathrm{i}}+\mathrm{w}\left(v_{i}, v^{\prime}\right)\right\}$

$v_{j} \in \overline{\mathrm{X}}_{k+1}$.If $u_{i}+w_{\mathrm{ij}}<u_{\mathrm{j}}$ then modify the original weights:

$\lambda_{j}=i$

$u_{\mathrm{j}}=u_{i}+w_{\mathrm{ij}}$

where,

$v^{\prime}$---the new intermediate point.

\subsection{Application Examples of Algorithm}

Fig. (1) is a typical industrial park. Now uses the Dijkstra algorithm to logistics scheduling.

The merchandise need to be transported from point (1) to point (6). Numbers on each line segment between two points are the distance value. The algorithm is as follows:

$u_{1}=0, u_{2}=20, u_{3}=6, \quad u_{4}=u_{5}=u_{6}=\infty$

$\lambda_{\mathrm{j}}=1 \quad(\mathrm{j}=2,3, \mathrm{~L}, 6)$

$X_{0}=\left\{v_{1}\right\}, \overline{X_{0}}=\left\{v_{2}, v_{3}, \mathrm{~L}, v_{6}\right\}$

$\min \left\{u_{2}, u_{3}, u_{4}, u_{5}, u_{6}\right\}=\{20,6, \infty, \infty, \infty\}=6=u_{3}$

$\lambda_{3}=1, X_{1}=\left\{v_{1}, v_{3}\right\}, \overline{X_{1}}=\left\{v_{2}, v_{4}, v_{5}, v_{6}\right\}$

$u_{2}=20, u_{3}+u_{32}=6+5=11$

$u_{3}+u_{32}<u_{2}, u_{4}=\infty, u_{3}+u_{34}=6+20=26$

$u_{5}=\infty, u_{3}+u_{35}=6+25=31, u_{3}+u_{35}<u_{5}$

Modify the temporary label:

$u_{2}=11, \lambda_{2}=3 ; u_{4}=26, \lambda_{4}=3 ; u_{5}=31, \lambda_{5}=3$

$\min \left\{u_{2}, u_{4}, u_{5}, u_{6}\right\}=\{11,26,31, \infty\}=11=u_{2}$

Make the point $v_{2}$ to get a permanent label:

$$
\begin{aligned}
& \lambda_{2}=3, X_{2}=\left\{\begin{array}{ll}
v_{1}, & v_{3}, v_{2}
\end{array}\right\}, \overline{X_{2}}=\left\{\begin{array}{ll}
v_{4}, v_{5}, v_{6}
\end{array}\right\} \\
& u_{2}+u_{24}=11+12=23<u_{4} \\
& u_{2}+u_{25}=u_{5}=31 \\
& u_{4}=23, \lambda_{4}=2 \\
& \min \left\{u_{4}, u_{5}, u_{6}\right\}=\{23,31, \infty\}=23=u_{4} \\
& \lambda_{4}=2, X_{3}=\left\{v_{1}, v_{3}, v_{2}, v_{4}\right\}, \overline{X_{3}}=\left\{v_{5}, v_{6}\right\} \\
& u_{5}=u_{4}+u_{45}=31, u_{4}+u_{46}=48<u_{6}, \lambda_{6}=4 \\
& \min \left\{u_{5}, u_{6}\right\}=\{31,48\}=31=u_{5}, \lambda_{5}=4 \\
& u_{5}+u_{56}=31+10=41=u_{6}, \lambda_{6}=5, \\
& X_{5}=\left\{v_{1}, v_{3}, v_{2}, v_{4}, v_{5}, v_{6}\right\}, \overline{X_{5}}=\Phi
\end{aligned}
$$

Ultimately, the algorithm ends. Finally the algorithm obtain the shortest path from (1) to (6) is (1) (3) (2) (4) (5) (6). Its length is 41 .

\section{THE IMPROVEMENT OF DIJKSTRA ALGO- RITHM}

From the above example, Dijkstra algorithm can easily find the shortest path when the starting and ending point is clear. However, the traditional method only takes into ac- 
count the length of the path, in actual industrial park logistics scheduling, choose the shortest route may not necessarily the best choice.

\subsection{The Main Idea of the Modified Algorithm}

There are many enterprises in the industrial park. The problem of the shortest path cannot be limited to the shortest path distance on a geographical sense. Because the theoretical shortest distance route is likely not in the actual delivery of the optimal path, suitable logistics scheduling should be to ensure its transportation time to be shortest. So it should take the actual running time of the selected roads as a major consideration in the logistics scheduling algorithm.

The main factors that affect the road running time are:

the weather;

$$
\text { the road traffic situation; }
$$

$$
\text { the vehicle performance. }
$$

According to the status of most domestic industrial parks, the author takes the road traffic as the most important factor that affects the delivery time. The author evaluates the running speed of motor vehicles to describe traffic state in the industrial parks. As showed below:

(1) roads open: not less than $30 \mathrm{~km} / \mathrm{h}$;

(2) mild crowding: $20-30 \mathrm{~km} / \mathrm{h}$;

(3) congestion: $10-20 \mathrm{~km} / \mathrm{h}$;

(4) blockage: less than $10 \mathrm{~km} / \mathrm{h}$.

By the definition of road traffic speed in real time, the article set an impact factor $\alpha$. It can change the weights of the roads that congestion occurs.

The definition of the value of $\mathrm{A}$ is as follows:

(1) roads open: $\alpha=1$;

(2) mild crowding: $\alpha=2$;

(3) congestion: $\alpha=4$;

$$
\text { blockage: } \alpha=\infty \text {. }
$$

Then multiply the impact factor by the path length to obtain the new weights.

\subsection{The Steps of Improved Algorithm}

Fig. (2) shows the abstract map of the park on the basis of traditional algorithm.

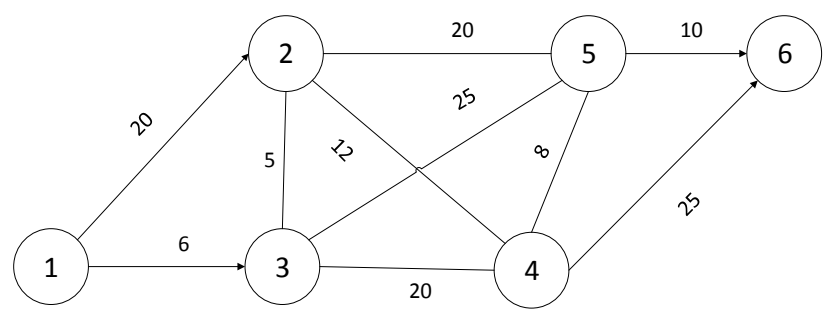

Fig. (2). The abstract map of Industrial Park.
In Fig. (2), arrows represent one-way traffic roads, straight lines represent two-way traffic roads and the numbers of segments represent the length of the road.

According to the detected real-time traffic conditions, the road between (1) and (3)、(2) and (4)、(2) and (5)、(5) and (6) are congested , road between (2) and (3)、(3) and (4) are mild crowded, and the rest are normal .

Fig. (3) is the new abstract map of changing the weights of the roads by impact factor $\alpha$.

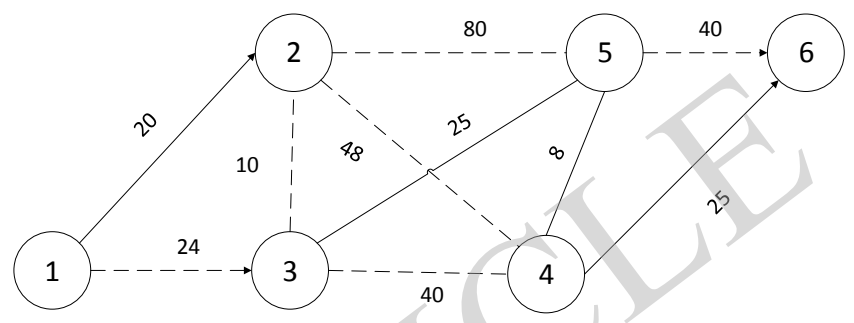

Fig. (3). The new abstract map.

Fig. (4) shows specific steps of the improved Dijkstra algorithm:

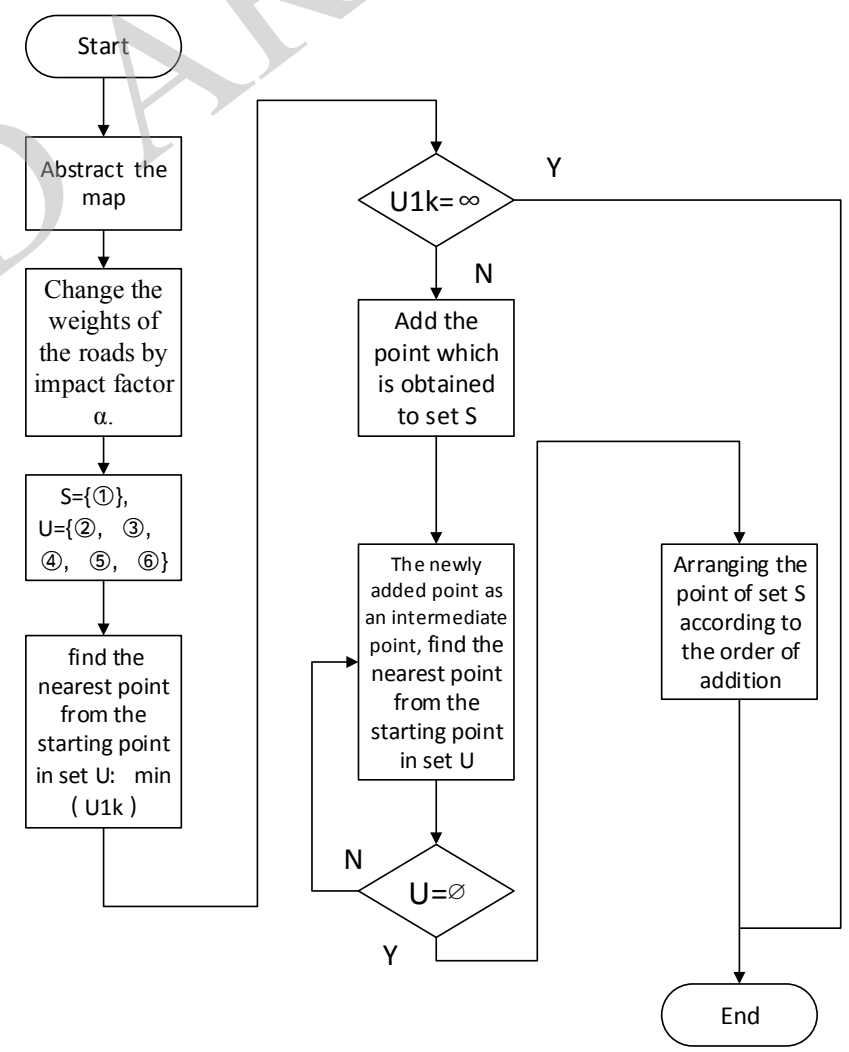

Fig. (4). The flowchart of new algorithm

In Fig. (4), every symbol is expressed as follows:

$S$---the set of points have been involved in operations;

$U$--- the set of points have not been involved in operations;

$U_{1 k}{ }^{---}$the shortest distance from the starting point. 
By the final calculation the shortest path from road (1) to (6) is (1) $\rightarrow$ (2) $\rightarrow$ (3) $\rightarrow$ (5) $\rightarrow$ (4) $\rightarrow$ (6), and its length is 83 .

\subsection{The Advantages of Improved Algorithm}

The improved Dijkstra algorithm processes and content is shown above. Contrast to the traditional Dijkstra algorithm, the new algorithm introduces the impact factor $\alpha$ which can change the weights according to the real-time road traffic conditions. So the new algorithm can choose the best driving roads under different traffic condition.

The comparison between improved algorithms and original algorithm is as follows:

The road between (1) and (3)、(2) and (4)、(2) and (5)、 (5) and (6) are congested. The average speed of these roads is $12 \mathrm{~km} / \mathrm{h} 、 12 \mathrm{~km} / \mathrm{h} 、 15 \mathrm{~km} / \mathrm{h} 、 10 \mathrm{~km} / \mathrm{h}$. The road between (2) and (3)、 (3) and (4) are mild crowded. The average speed of these roads is $25 \mathrm{~km} / \mathrm{h}$. The remaining roads are in normal situation, the average speed is $35 \mathrm{~km} / \mathrm{h}$.

Weights from Fig. (2) represent the actual distance and the unit is kilometer. So the distance from (1) to (3) is $6 \mathrm{~km}$, and (1) to (2) is $20 \mathrm{~km}$, and so on.

The path which is calculated by traditional algorithm is (1) (3) (2) (4) (5) (6). The total length is $41 \mathrm{~km}$, and the delivery time according to the real-time speed is:

$$
\frac{6}{12}+\frac{5}{25}+\frac{12}{12}+\frac{8}{35}+\frac{10}{10}=\frac{13}{14}+2 \approx 2.928 h
$$

The path which is calculated by improved algorithm is (1) (2) (3) (5) (4) (6).The total length is $83 \mathrm{~km}$, and the delivery time according to the real-time speed is:

$$
\frac{20}{35}+\frac{5}{25}+\frac{25}{35}+\frac{8}{35}+\frac{25}{35}=\frac{3}{7}+2 \approx 2.428 h
$$

Judge from the two algorithms above, the delivery time of the improved Dijkstra algorithm is less than the original algorithm although the distance appears to be twice than the first algorithm. In fact, it can avoid the congested roads and reach the destination earlier. More importantly, the improved algorithm bypasses some road sections which may have further serious road congestion so that it can avoid the losses of the enterprise that caused by logistics vehicles blocking.

\section{CONCLUSION}

This paper research on the optimization problem of logistics scheduling of the industrial park, mainly studied the Dijkstra shortest path algorithm and improvements. Analyze and improve the limitations of existing Dijkstra algorithm. By comparison of the two algorithms, the improved algorithm is real-time. It can adapt to different roads in different situations, with strong scientific rationality and practicality. Make a reasonable use of the improved Dijkstra algorithm, it can greatly reduce the original distribution costs, avoid losses related businesses because of traffic problems caused. Former industrial park logistics scheduling problems can get a better solution. If the algorithm is widely used in the industrial park, it will play out more advantages to put the park logistics scheduling to a new height.

\section{CONFLICT OF INTEREST}

The authors confirm that this article content has no conflict of interest.

\section{REFERENCES}

[1] F. Xue, "Development and status of the logistics center". Modern Logistics, no. 3, pp. 22-23, 2010.

[2] T. Yu, and D. Zhu, "The research of logistics alliance formation mechanism". Logistics Technology, no. 2, pp. 34-36, 2009.

[3] X. Zhuang, Y. Cai, H. Zhu, and D. Zhou, "Stability analysis method of Rock slope based on the Meshless - Graph Theory". Chinese Quarterly of Mechanics, vol. 29, no.4, pp. 537-543, 2008.

[4] S. Li, "Transportation Network Modeling Study Variables and the Research of Shortest Path Algorithm Based on Petri Net,". Guangxi Normal University, 2012.

[5] $\quad$ Feng Z. "The Design and Research of Three-Dimensional Digital Campus System Based on GIS,”. Dalian Maritime University, 2013. 